\title{
Predictive factors of complications and 30-day mortality in patients undergoing percutaneous endoscopic gastrostomy: the utility of C-reactive protein to albumin ratio
}

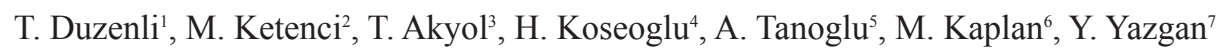 \\ (1) Department of Gastroenterology, Hitit University Erol Olcok Training and Research Hospital, Corum, Turkey ; (2) Department of Internal Medicine, Sultan \\ Abdulhamid Han Training and Research Hospital, Istanbul, Turkey ; (3) Department of Gastroenterology, Samsun Gazi State Hospital, Samsun, Turkey ; (4) Department \\ of Gastroenterology, Hitit University Erol Olcok Training and Research Hospital, Corum, Turkey ; (5) Department of Gastroenterology, Sultan Abdulhamid Han Training \\ and Research Hospital, Istanbul, Turkey ; (6) Department of Internal Medicine, Sultan Abdulhamid Han Training and Research Hospital, Istanbul, Turkey ; (7) Department \\ of Gastroenterology, Sultan Abdulhamid Han Training and Research Hospital, Istanbul, Turkey.
}

\begin{abstract}
Background and study aims : Percutaneous endoscopic gastrostomy (PEG) is a procedure that provides long term enteral nutrition. To investigate the predictors of PEG-related complications and 30-day mortality rates and evaluate the indicators for deciding whether to recommend elective PEG insertions, we sought to determine the complications and early mortality rates of patients who underwent PEG.

Patients and methods: We performed a retrospective analysis of consecutive adult patients who had undergone PEG for the first time between October 2016 and January 2019. The predictors of complications and 30-day mortality were analyzed with receiver operating characteristic (ROC) and logistic regression analysis.

Results: This study included 309 patients. Patients were excluded from the study if they were $<18$ years of age or there were missing data about them. Out of 253 patients, $33(13 \%)$ had complications and $32(12.6 \%)$ died within one month after PEG insertion. A higher C-reactive protein (CRP) to albumin ratio was the only independent factor predicting the complications (odds ratio $(\mathrm{OR}): 3.17 ; 95 \% \mathrm{CI}: \mathbf{1 . 2 6 - 8 . 0 0} ; \mathrm{p}=\mathbf{0 . 0 1 4})$. The independent predictive factors for 30-day mortality after PEG placement included higher urea levels and higher CRP to albumin ratios $(O R$ : $3.78 ; 95 \%$ CI $: 1.41-10.17 ; p=0.008)($ OR : $6.67 ; 95 \%$ CI : $1.87-$ $23.75 ; p=0.003)$. The only predictor for both complications and 30-day mortality was the CRP to albumin ratio.

Conclusions: When appropriate, the PEG procedure can provide a safe and effective method for enteral feeding. The CRP to albumin ratio can be used to predict complications and early mortality after PEG insertion. Because PEG is elective, higher CRP to albumin ratios can be helpful in deciding to select patients for the procedure. (Acta gastroenterol. belg., 2021, 84, 283-288).
\end{abstract}

Keywords: CAR, C-reactive protein, CRP to albumin ratio, percutaneous endoscopic gastrostomy, predictor.

\section{Introduction}

Percutaneous endoscopic gastrostomy (PEG) is the preferred method for administering long-term enteral nutrition to patients who require prolonged feeding tube support (1). Although it is a minimally invasive and relatively safe procedure, it can result in adverse events. To illustrate, post-procedural complication rates and short-term mortality for PEG are $13.2 \%-42.9 \%$ and $3.3 \%-23.9 \%$, respectively $(2-5)$.

In addition to addressing patients' long-term nutritional needs, it is also important to assess patients' clinical status and life expectancy when deciding to insert PEG tube. Patients considered for PEG may be at increased risk of complications due to malnutrition and comorbidities, such as diabetes, cardiovascular diseases, and cancer. As there are alternatives to PEG placement that may be more appropriate for high-risk patients, it is critical to understand the risk factors that can lead to poor outcomes during patient selection and the decisionmaking process.

Although some studies in the literature have predicted the complication and mortality rates that are associated with PEG, there remains a need for fast, effective, inexpensive, and easily accessible markers. This study aimed to determine the complications and short term mortality in patients undergoing PEG in our tertiary reference center hospital, to investigate the predictive factors of complications and 30-day mortality, and to evaluate these indicators for making the decision for elective PEG insertion.

\section{Patients and methods}

We performed a retrospective analysis of consecutive adult patients who underwent PEG insertions for the first time at the Health Science University Sultan Abdulhamid Han Training and Research Hospital between October 2016 and January 2019. Patients were excluded from the study if they had missing records, were $<18$ years of age, or could not have a PEG tube inserted for any reason. The local ethics committee of Health Science University approved the study. Demographic data, clinical conditions, indications, requesting departments, and the patients' comorbidities were obtained from the hospital computer system. Complete blood count, urea, creatinine, sodium, potassium, albumin, and C-reactive protein (CRP) tests were performed for all patients before the procedure. PEG tubes were inserted using the pull-through method in patients who had not fed orally

Correspondence to : Tolga Düzenli, M.D., Department of Gastroenterology, Hitit University Erol Olcok Training and Research Hospital, Corum, Turkey. Phone : +90 (364) 21930 00. Fax. : + 90 (364) 2193030.

E-mail: tolgaduzenli@yahoo.com

Submission date : 05/06/2020

Acceptance date : 22/08/2020 
Table 1. - Demographic and clinical characteristics of patients with PEG insertion

\begin{tabular}{|c|c|c|c|}
\hline & & $\mathrm{n}=\mathbf{2 5 3}$ & $\%$ \\
\hline \multicolumn{4}{|c|}{ Age } \\
\hline & $\begin{array}{lll}\text { Between } & 26-104 \quad \text { (mean: } \\
78,1 \pm 12,2) & \end{array}$ & & \\
\hline \multicolumn{4}{|c|}{ Sex } \\
\hline & Female & 140 & 55.3 \\
\hline & Male & 113 & 44.7 \\
\hline \multicolumn{4}{|c|}{ Requesting department } \\
\hline & Neurology & 64 & 25.3 \\
\hline & Pulmonary diseases & 71 & 28.1 \\
\hline & Internal Medicine & 87 & 34.4 \\
\hline & Intensive care & 17 & 6.7 \\
\hline & Palliative care & 1 & 0.4 \\
\hline & Neurosurgery & 5 & 2 \\
\hline & Emergency Department & 3 & 1.2 \\
\hline & Ear-nose-throat & 5 & 2 \\
\hline \multicolumn{4}{|c|}{ Indication of PEG } \\
\hline & Dementia & 150 & 59.3 \\
\hline & Dysphagic stroke & 60 & 23.7 \\
\hline & Advanced Parkinsonism & 12 & 4.7 \\
\hline & Trauma & 6 & 2.4 \\
\hline & Motor neurone disease & 6 & 2.4 \\
\hline & Multiple sclerosis & 3 & 1.2 \\
\hline & Hypoxic encephalopathy & 6 & 2.4 \\
\hline & Head and neck cancers & 10 & 4 \\
\hline \multicolumn{4}{|c|}{ Charlson comorbidity index } \\
\hline & Between 0-12 (mean: $4.8 \pm 2.0$ ) & & \\
\hline \multicolumn{4}{|c|}{ Complication } \\
\hline & No & 220 & 87 \\
\hline & Yes & 33 & 13 \\
\hline & Tube leakage & 11 & 4.3 \\
\hline & Tube dislodgement & 10 & 4 \\
\hline & Wound infection & 6 & 2.4 \\
\hline & Tube blockage & 2 & 0.8 \\
\hline & Buried bumper syndrome & 2 & 0.8 \\
\hline & Aspiration & 2 & 0.8 \\
\hline \multicolumn{4}{|c|}{ Mortality (30-day) } \\
\hline & No & 221 & 87.4 \\
\hline & Yes & 32 & 12.6 \\
\hline
\end{tabular}

for at least six weeks and whose life expectancies were evaluated by their physicians. Most of the procedures were performed using local anesthesia with lidocaine. For agitated patients, sedoanalgesia was given as intravenous (IV) midazolam and IV pethidine.

The procedures were performed with a standard gastroscope device (Fujinon EG-590 WR). All patients received prophylactic antibiotics (e.g., cefazoline) 30 minutes before the PEG process began. In accordance with recommended guidelines, patients using anticoagulants were managed with a necessary cessation period (6). Oxygen saturation, pulse rate, and blood pressure were monitored during the PEG insertion. First, the patients underwent a control endoscopy. Then, a $20 \mathrm{Fr}$ removable PEG tube (Boston Scientific, Boston, MA, USA) was placed on the anterior wall of the corpus. Written consent was routinely obtained from the patients or their representatives before the procedure and after information about PEG placement and its associated complications had been provided. Physical examinations were performed four hours after the procedure had taken place. Patients without abdominal discomfort were initially given low doses of enteral feeding and liquids. Doses for enteral feeding were gradually increased within 24 hours, and routine feeding was optimized the following day. Complications and deaths that occurred within 30 days of the procedure were recorded and analyzed.

The patients' comorbidities were reviewed, and the Charlson Comorbidity Index - which includes 20 variables of diseases - was calculated for the statistical analysis of each patient. Statistical analysis was performed using SPSS software (Statistical Package for the Social Sciences, version 15.0, SSPS Inc., Chicago, IL, USA). The study group's descriptive statistics (percentage, mean, median, and distributions) were then determined. A student's $t$ test was used for normally distributed parameters, and the Mann-Whitney U test was used for parameters that were not normally distributed. Categorical data were investigated with a chi-square test. The cut-off values were calculated using receiver operating characteristic (ROC) analysis. The predictive factors for complications and 30-day mortality were determined with logistic regression analysis. A value of $p$ $<0.05$ was considered to be statistically significant.

\section{Results}

This study included a total of 309 patients. Patients who had missing records or were $<18$ years of age were excluded from the study. The data for 253 patients were investigated retrospectively. The demographic and clinical characteristics of the patients are presented in Table 1. The patients were between 26-104 years old (mean : $78.1 \pm 12.2$ ), and $55.3 \%$ of them were women. The most common indication for PEG insertion was dementia (59.3\%), and the most frequent clinic to request PEG was neurology (25.3\%). The Charlson Comorbidity Index ranged between $0-12$ (mean : $4.8 \pm 2.0$ ).

Complications were observed in $33(13 \%)$ of the 253 patients. The most common complications included tube leakage $(\mathrm{n}=11 ; 4.3 \%)$, tube dislodgement $(\mathrm{n}=10 ; 4 \%)$, and wound infection $(\mathrm{n}=6 ; 2.4 \%)$. Other complications included tube blockage ( $\mathrm{n}=2 ; 0.8 \%)$, buried bumper syndrome $(\mathrm{n}=2 ; 0.8 \%)$, and aspiration $(\mathrm{n}=2 ; 0.8 \%)$. Although $32(12.6 \%)$ patients died within 30 days of PEG insertion, none of the patients died due to PEG complications. No major life-threatening complications were observed in any of the patients.

Table 2 presents the clinical and laboratory features of the patients with and without complications and 30day mortality. When compared to the group of patients without complications, the CRP and CRP to albumin ratios were higher in patients with complications $(\mathrm{p}<$ 0.013 and $\mathrm{p}<0.018$ ). 
Table 2. - Clinical and laboratory features of patients with and without complications and 30-day mortality

\begin{tabular}{|c|c|c|c|c|c|c|}
\hline & \multicolumn{3}{|c|}{ Complications } & \multicolumn{3}{|c|}{ 30-day mortality } \\
\hline & Yes & No & $\mathrm{p}$ & Yes & No & $\mathrm{p}$ \\
\hline Age, years & $78.9 \pm 12.7$ & $77.8 \pm 12.3$ & 0.455 & $79.8 \pm 12.9$ & $77.7 \pm 12.3$ & 0.201 \\
\hline Sex, female & $21 / 33,64 \%$ & $119 / 220,54 \%$ & 0.304 & $22 / 32,69 \%$ & $118 / 221,53 \%$ & 0.102 \\
\hline BMI, $\mathrm{kg} / \mathrm{m}^{2}$ & $22.88 \pm 6.94$ & $23.56 \pm 6.08$ & 0.253 & $23.34 \pm 6.67$ & $23.67 \pm 5.26$ & 0.498 \\
\hline Charlson comorbidity index & $5.2 \pm 2.2$ & $4.8 \pm 2$ & 0.163 & $5.4 \pm 2$ & $4.8 \pm 2$ & $0.047^{*}$ \\
\hline $\mathrm{WBC}, 10^{3} / \mu \mathrm{L}$ & $8.7 \pm 2.7$ & $8.9 \pm 3.6$ & 0.729 & $9.3 \pm 3.3$ & $8.8 \pm 3.5$ & 0.481 \\
\hline Hemoglobin, g/dL & $11.2 \pm 1.8$ & $10.9 \pm 1.7$ & 0.270 & $10.2 \pm 1.4$ & $11.0 \pm 1.8$ & $0.007^{*}$ \\
\hline MCV, fL & $89.2 \pm 5.1$ & $88.1 \pm 5.3$ & 0.247 & $88.6 \pm 4$ & $88.2 \pm 5.5$ & 0.639 \\
\hline RDW, \% & $13.6 \pm 1.7$ & $14.3 \pm 2.2$ & 0.069 & $15 \pm 2.1$ & $14.1 \pm 2.2$ & $0.005^{*}$ \\
\hline Platelets, $10^{3} / \mu \mathrm{L}$ & $263.7 \pm 112.3$ & $244.7 \pm 92.9$ & 0.564 & $224.4 \pm 81$ & $250.6 \pm 97.3$ & 0.118 \\
\hline MPV, fL & $7.7 \pm 1.5$ & $8.3 \pm 1.6$ & 0.051 & $8.3 \pm 1.6$ & $8.27 \pm 1.6$ & 0.550 \\
\hline Neutrophils, $10^{3} / \mu \mathrm{L}$ & $6.2 \pm 2.6$ & $6.4 \pm 3.1$ & 0.595 & $7.1 \pm 3.0$ & $6.3 \pm 3.1$ & 0.112 \\
\hline Lymphocyte, $10^{3} / \mu \mathrm{L}$ & $1.7 \pm 0.7$ & $1.7 \pm 0.9$ & 0.497 & $1.4 \pm 0.9$ & $1.7 \pm 0.9$ & $0.011 *$ \\
\hline C-reactive protein, $\mathrm{mg} / \mathrm{L}$ & $64.3 \pm 43.1$ & $45.6 \pm 37.0$ & $0.013 *$ & $68.1 \pm 39.2$ & $45,0 \pm 37,3$ & $<0.001^{*}$ \\
\hline Albumin, g/dl & $2.8 \pm 0.4$ & $2.9 \pm 0.6$ & 0.809 & $2,7 \pm 0,5$ & $2.9 \pm 0.8$ & $0.042 *$ \\
\hline Sodium, mmol/L & $136.6 \pm 7.1$ & $138 \pm 6.2$ & 0.164 & $138.1 \pm 7.4$ & $137.8 \pm 6.1$ & 0.938 \\
\hline Potassium, $\mathrm{mmol} / \mathrm{L}$ & $4.1 \pm 0.7$ & $3.9 \pm 0.6$ & 0.266 & $3.9 \pm 0.7$ & $4.0 \pm 0.6$ & 0.560 \\
\hline Urea, mg/dL & $51.8 \pm 35.5$ & $54.8 \pm 42.4$ & 0.944 & $83.7 \pm 65.6$ & $45.4 \pm 34.8$ & $<0.001^{*}$ \\
\hline Creatinine, $\mathrm{mg} / \mathrm{dL}$ & $0.90 \pm 0.33$ & $0.93 \pm 0.49$ & 0.947 & $1.07 \pm 0.58$ & $0.91 \pm 0.45$ & 0.111 \\
\hline CRP to albumin ratio & $23.7 \pm 16.9$ & $17.1 \pm 15.2$ & $0.018^{*}$ & $27.1 \pm 18.8$ & $16.6 \pm 14.5$ & $<0.001 *$ \\
\hline NLR & $4.3 \pm 2.7$ & $6.4 \pm 19.2$ & 0.524 & $7.3 \pm 5.9$ & $6.0 \pm 19.1$ & $0.003 *$ \\
\hline PLR & $172.9 \pm 87$ & $248.5 \pm 921.3$ & 0.995 & $226.3 \pm 222.4$ & $240.6 \pm 918.4$ & 0.208 \\
\hline
\end{tabular}

BMI, body mass index ; WBC, white blood cells ; MCV, mean corpuscular volume ; RDW, redcell distribution width ; MPV, mean platelet volume ; CRP, c-reactive protein ; NLR, neutrophil to lymphocyte ratio ; PLR, platelet to lymphocyte ratio. ${ }^{*} \mathrm{p}<0.05$ was considered statistically significant.

The Charlson Comorbidity Index $(\mathrm{p}=0.047)$, red cell distribution width (RDW) $(\mathrm{p}=0.005), \mathrm{CRP}(\mathrm{p}<0.001)$ urea $(\mathrm{p}<0.001)$, CRP to albumin ratio $(\mathrm{p}=0.001)$, and neutrophil to lymphocyte ratio $(\mathrm{NLR})(\mathrm{p}=0.003)$ were significantly higher in the deceased patients than in the survivors. The hemoglobin $(\mathrm{p}=0.007)$, lymphocytes $(\mathrm{p}=0.011)$, and albumin $(\mathrm{p}=0.042)$ values were significantly lower in the deceased patients than in the survivors. No relationships were observed between the
Table 3. - Chi-square test between complications and 30-day mortality

\begin{tabular}{|c|c|c|c|c|c|}
\hline & \multicolumn{2}{|c|}{ Mortality } & \multirow{2}{*}{ Total } & \multirow{2}{*}{$\mathrm{p}^{*}$} \\
\hline & & No & Yes & & \\
\hline \multirow{2}{*}{ Complications } & No & 194 & 26 & 220 & \multirow{3}{*}{0,305} \\
\hline & Yes & 27 & 6 & 33 & \\
\hline \multicolumn{2}{|l|}{ Total } & 221 & 32 & 253 & \\
\hline
\end{tabular}

$* \mathrm{p}<0.05$ was considered statistically significant.
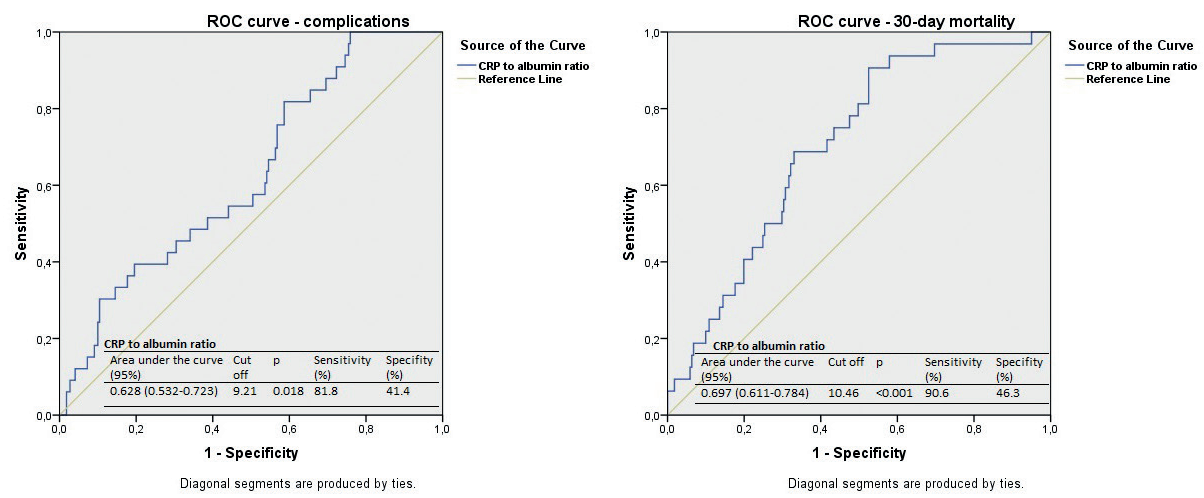

Figure 1. - Receiver operating characteristic (ROC) analysis of CRP to albumin ratio for complications and 30-day mortality after PEG placement. 
Table 4. - Receiver operating characteristic (ROC) analysis of parameters associated with complications and 30-day mortality after PEG placement

\begin{tabular}{|c|c|c|c|c|c|}
\hline & AUC $(95 \%)$ & Cut off value & $\mathrm{p}$ & $\begin{array}{c}\text { Sensitivity } \\
(\%)\end{array}$ & Specifity (\%) \\
\hline \multicolumn{6}{|l|}{ Complications } \\
\hline - $\mathrm{CRP}$ to albumin ratio & $0.628(0.532-0.723)$ & 9.21 & $0.018 *$ & 81.8 & 41.4 \\
\hline \multicolumn{6}{|l|}{ 30-day mortality } \\
\hline - Charlson comorbidity index & $0.603(0.498-0.709)$ & 4.5 & 0.059 & 62.5 & 50 \\
\hline - Hemoglobin, g/dL & $0.356(0.256-0.457)$ & 9.74 & $0.009 *$ & 56.3 & 25 \\
\hline - RDW, \% & $0.650(0.543-0.756)$ & 13.65 & $0.006^{*}$ & 75 & 45.8 \\
\hline - Lymphocyte, $10^{3} / \mu \mathrm{L}$ & $0.363(0.253-0.473)$ & 1.3 & $0.013^{*}$ & 53.1 & 34.3 \\
\hline - Urea, mg/dL & $0.675(0.578-0.773)$ & 37.5 & $0.001 *$ & 81.3 & 44 \\
\hline - CRP to albumin ratio & $0.697(0.611-0.784)$ & 10.46 & $<0.001 *$ & 90.6 & 46.3 \\
\hline - NLR & $0.663(0.561-0.765)$ & 3.6 & $0.003 *$ & 71.9 & 50 \\
\hline
\end{tabular}

AUC, area under the curve ; RDW, red cell distribution width ; CRP, C-reactive protein ; NLR, neutrophil to lymphocyte ratio. * $\mathrm{p}<0.05$ was considered statistically significant.

groups with complications and 30-day mortality $(\mathrm{p}=$ 0.305 ) (Table 3). Although six of the deceased patients also had complications, none of these patients died due to complications.

Cut-off values were determined using Youden's index by ROC curve analysis (Table 4 ; Figure 1). The parameters associated with complications and 30-day mortality after PEG insertion were investigated using a logistic regression analysis with the cut-off values defined by the ROC curve analysis (Table 5). The CRP to albumin ratio appeared to predict complications (odds ratio $(\mathrm{OR}): 3.783 ; 95 \% \mathrm{CI}: 1.407-10.171 ; \mathrm{p}=0.008)$ and 30-day mortality (OR: 6.670 ; 95\% CI : 1.873$23.752 ; p=0.003)$. Urea was the only other factor that predicted mortality (OR : $3.783 ; 95 \% \mathrm{CI}: 1.407$ $10.171 ; p=0.008)$. The CRP, Charlson Comorbidity Index, hemoglobin, RDW, lymphocytes, albumin, and NLR were not statistically significant predictors of mortality $(\mathrm{p}>0.05)$.

\section{Discussion}

For patients who require long-term nutritional support, PEG is one of the most common and preferred methods for providing enteral nutrition (1). In the present study, one of our aims was to determine the complications and 30-day mortality in patients who had undergone PEG insertion. Although PEG is known to be safe, the procedure can also result in adverse outcomes. According to the literature, the complication rates of PEG insertion have been reported to range from $13.2 \%$ to $42.9 \%$ (25). In addition, the frequency of 30-day mortality has been reported to range from $2.4 \%$ to $22.5 \%(4-5,7)$. Although the overall mortality rate is high for patients who undergo PEG insertion - especially in patients with severe comorbidities - PEG-related mortality is low (8). The complication rate for the patients in the present study was $13 \%$ and the 30 -day mortality rate was
$12.6 \%$. These findings are compatible with observations from the literature. Blomberg et al. reported an $18 \%$ rate mortality in a period of 2 months after PEG insertion (9). Schneider et al. observed a $10 \%$ rate of mortality within a timeframe of 30 days, which was not directly correlated to the PEG procedure (10).

The most common complications examined in the current study included tube leakage, tube dislodgement, and wound infection, which is in line with findings from the literature (7-12). The most frequently reported complications in the literature included leakage (up to $78 \%$ ), peristomal infection (up to $32 \%$ ), and early mortality ( $8 \%-28 \%$ mortality within 30 days of PEG insertion) (9). Complications can be classified according to severity (minor vs. major) or time of occurrence (12). The vast majority of complications observed in the present study were minor complications. Hucl et al. reported that while most of the complications that occurred in their study were minor, their incidence was not negligible (12).

As with other studies in the literature, neurological diseases were the most common reason that PEG was recommended $(2,4,11)$. In as many as $90 \%$ of cases, a reduction in oral intake due to neurodegenerative processes is the primary reason that patients undergo PEG placement (7-11). One interesting finding from this study was that dementia - which is a controversial indication for PEG tube placement - was the major indication for PEG placement (59\%). In Lee et al.'s series of seven tertiary hospitals in the Republic of Korea, only $4 \%$ of patients suffered from dementia (4). It is our belief that cultural differences may explain this discrepancy.

Comorbidities were calculated with the Charlson Comorbidity Index. The index consists of 20 different disease group variables and is widely used in research due to its simple structure and its ability to facilitate easier patient evaluations $(11,13)$. Shangab et al. observed that low albumin and a high Charlson Comorbidity 
Index were predictors of adverse events for patients who had undergone PEG insertions (11). In the present study, although the Charlson Comorbidity Index was significantly higher in deceased patients than it was in survivors, the index was not a predictor of mortality or complications in the logistic regression analysis.

In light of these findings, it is important to identify the patients who would maximally benefit from PEG insertion (12). Albumin and CRP levels have previously been investigated as predictors of early mortality and acute conditions $(4-5,7,14-15)$. Lee et al. reported that CRP values above $21.5 \mathrm{mg} / \mathrm{L}$ and albumin values below $3.15 \mathrm{~g} / \mathrm{dL}$ increased mortality. They also noted that if both of these parameters were present in the same patient, the median survival time significantly decreased (4). Bloomberg et al. expressed that CRP levels higher than $10 \mathrm{mg} / \mathrm{L}$ and albumin values lower than $3 \mathrm{~g} / \mathrm{dL}$ were independent risk factors for post-PEG mortality (5). In a study by Tominaga et al. that included 84 patients, the authors reported that low albumin was a critical risk factor in 1-year mortality after PEG insertion (16).

The current study revealed that a higher CRP to albumin ratio resulted in a 3.2-fold increase in complications and a 6.7-fold increase in 30-day mortality. High CRP and low albumin ratios have also been proven to act as markers for several conditions, such as inflammation, postoperative infection, and malnutrition (5). In addition, the CRP to albumin ratio has been reported to function as a marker for many inflammatory, malignant, and infectious diseases (17). For these reasons, the CRP to albumin ratio has drawn attention as a predictive factor for complications and 30-day mortality. The CRP to albumin ratio also has the advantage of being cheap, fast, and easy to use. Because PEG is an elective procedure, we believe that it is important to avoid or delay it in patients with high CRP to albumin ratios and to treat the underlying causes that produce these increased levels. In Barbosa et al.'s investigation of the predictive role of CRP, they stated that it would be appropriate to postpone PEG procedures in patients with high CRP, especially during the early period. The authors also suggested that information be provided to patients' families and less invasive and alternative methods for feeding be recommended (18).

Another important result of the present study was the predictive role that higher urea levels play in 30day mortality. To the best of our knowledge, no study in the literature has investigated the predictive value of higher urea for the mortality rates of patients who undergo PEG insertion. Nonetheless, some studies have indicated that urea levels can be used to predict shortterm mortality rates in cardiac diseases (19). Moreover, Sbeit et al. indicated that creatinine and some other parameters could predict short-term mortality after PEG (20). In the current study, although no differences were observed between the groups' creatinine values, urea was identified as an independent predictive factor for 30-day mortality after PEG placement.
The NLR was significantly higher in this study's deceased patients than in its survivors. However, the significance of this finding decreased during the logistic regression analysis. Indeed, it is well known that the NLR can be elevated under the conditions of several diseases $(21,22)$. As in other studies in the literature, the sensitivity and specificity of the NLR remained low in the current study.

This study contained certain limitations. The first and most significant of these limitations was the study's retrospective design. Although patients with missing data were eliminated, there is the potential for data about adverse events to be misinterpreted due to the study's retrospective nature. Second, the data does not reflect the characteristics of the general population since the study was conducted at a single center. Nevertheless, because the hospital in this study functions as a tertiary reference center for the region, we believe that the present study's patient profile covers most of the relevant population.

In conclusion, PEG provides a safe and effective method for enteral feeding in qualified patients. This study indicated that the CRP to albumin ratio can be used to predict complications and early mortality rates after PEG insertion. Because PEG is an elective procedure, higher CRP to albumin ratios may prove helpful in selecting patients and deciding if they are suitable for the PEG procedure.

\section{References}

1. ANDERLONI A., DI LEO M., BARZAGHI F., SEMERARO R., MEUCCI G., MARINO R., et al. Complications and early mortality in percutaneous endoscopic gastrostomy placement in lombardy : A multicenter prospective cohort study. Dig. Liver Dis., 2019, 51(10) : 1380-1387.

2. PIH G.Y., NA H.K., AHN J.Y., JUNG K.W., KIM D.H., LEE J.H., et al. Risk factors for complications and mortality of percutaneous endoscopic gastrostomy insertion. BMC Gastroenterol., 2018, 18(1) : 101.

3. VUJASINOVIC M., INGRE C., BALDAQUE SILVA F., FREDERIKSEN F., YU J., ELBE P. Complications and outcome of percutaneous endoscopic gastrostomy in a high-volume centre. Scand. J. Gastroenterol., 2019, 54(4) : 513-518.

4. LEE C., IM J.P., KIM J.W., KIM S.E., RYU D.Y., CHA J.M., et al. Risk factors for complications and mortality of percutaneous endoscopic gastrostomy : a multicenter, retrospective study. Surg. Endosc., 2013, 27(10) : 3806-3815.

5. BLOMBERG J., LAGERGREN P., MARTIN L., MATTSSON F., LAGERGREN J. Albumin and C-reactive protein levels predict short-term mortality after percutaneous endoscopic gastrostomy in a prospective cohort study. Gastrointest. Endosc., 2011, 73(1) : 29-36.

6. ASGE STANDARDS OF PRACTICE COMMITTEE, ACOSTA R.D., ABRAHAM N.S., CHANDRASEKHARA V., CHATHADI K.V., EARLY D.S., et al. The management of antithrombotic agents for patients undergoing GI endoscopy [published correction appears in Gastrointest Endosc. 2016 Mar;83(3):678]. Gastrointest. Endosc., 2016, 83(1) : 3-16.

7. KARASAHIN O., TASAR P.T., TIMUR O., BINICI D.N., YILMAZ T.K., ASLAN A., et al. High C-Reactive Protein and Low Albumin Levels Predict High 30-Day Mortality in Patients Undergoing Percutaneous Endoscopic Gastrotomy. Gastroenterology Res., 2017, 10(3) : 172-176.

8. ZOPF Y., MAISS J., KONTUREK P., RABE C., HAHN E.G., SCHWAB D. Predictive factors of mortality after PEG insertion: guidance for clinical practice. JPEN J. Parenter. Enter. Nutr., 2011, 35 : 50e5.

9. BLOMBERG J., LAGERGREN J., MARTIN L., MATTSSON F., LAGERGREN P. Complications after percutaneous endoscopic gastrostomy in a prospective study. Scand. J. Gastroenterol., 2012, $47: 737 \mathrm{e} 42$.

10. SCHNEIDER A.S., SCHETTLER A., MARKOWSKI A., LUETTIG B., KAUFMANN B., KLAMT S., et al. Complication and mortality rate after percutaneous endoscopic gastrostomy are low and indication-dependent. Scand. J. Gastroenterol., 2014, 49 : 891e8. 
11. SHANGAB M.O.M., SHAIKH N.A. Prediction of risk of adverse events related to percutaneous endoscopic gastrostomy: a retrospective study. Ann. Gastroenterol., 2019, 32(5) : 469-475.

12. HUCL T., SPICAK J. Complications of percutaneous endoscopic gastrostomy. Best. Pract. Res. Clin. Gastroenterol., 2016, 30(5) : 769-781.

13. QUAN H., LI B., COURIS C.M., FUSHIMI K., GRAHAM P., HIDER P., et al. Updating and validating the Charlson comorbidity index and score for risk adjustment in hospital discharge abstracts using data from 6 countries. Am.J. Epidemiol., 2011, 173 : 676-682.

14. MARTIN L., LAGERGREN J., BLOMBERG J., JOHAR A., BOSAEUS I., LAGERGREN P. Phase angle as a prognostic marker after percutaneous endoscopic gastrostomy (PEG) in a prospective cohort study. Scand. J. Gastroenterol., 2016, 51(8) : 1013-1016.

15. HIGAKI F., YOKOTA O., OHISHI M. Factors predictive of survival after percutaneous endoscopic gastrostomy in the elderly: is dementia really a risk factor? Am. J. Gastroenterol., 2008, 103(4) : 1011-1016; quiz 1017.

16. TOMINAGA N., SHIMODA R., IWAKIRI R., TSURUOKA N., SAKATA Y., HARA H., et al. Low serum albumin level is risk factor for patients with percutaneous endoscopic gastrostomy. Intern. Med., 2010, 49(21) : 2283-2288.
17. OH T.K., SONG I.A., LEE J.H. Clinical usefulness of C-reactive protein to albumin ratio in predicting 30 -day mortality in critically ill patients: A retrospective analysis. Sci. Rep., 2018, 8(1) : 14977.

18. BARBosa M., MAGAlHAES J., MARINHO C., COTTER J. Predictive factors of early mortality after percutaneous endoscopic gastrostomy placement : The importance of C-reactive protein. Clin. Nutr. ESPEN., 2016, 14 : 19-23.

19. KHOURY J., BAHOUTH F., STABHOLZ Y., ELIAS A., MASHIACH T., ARONSON D., et al. Blood urea nitrogen variation upon admission and at discharge in patients with heart failure. ESC Heart Fail., 2019, 6(4) : 809816.

20. SBEIT W, KADAH A, MARI A, MAHAMID M, KHOURY T. Simple Bedside Predictors of Survival after Percutaneous Gastrostomy Tube Insertion. Can. J. Gastroenterol. Hepatol., 2019, 2019 : 1532918. Published 2019 Nov 16.

21. DUZENLI T., KOSEOGLU H. NLR as a prognostic marker in metastatic gallbladder cancer? HPB (Oxford), 2020, 22(5) : 793

22. TANOGLU A., DUZENLI T. Neutrophil-to-lymphocyte ratio alone may not be a true indicator of the severity of acute pancreatitis. Turk. J. Gastroenterol., 2019, 30(10) : 937 